\title{
Smart Wheel Chair to Assist Physically Challenged
}

\author{
N.Monicka, Bhuvaneswari Balachander
}

\begin{abstract}
Paper describes a smart, key and a motor controlled wheel chair. This is been designed for the disabled persons and aged ones. It is a "Key-controlled Wheel chair" that follows the commands given. The commands are given by a smart phone which has Bluetooth and the command is transmitted to the Line follower Arduino board. For example, when the user gives command like "Go to room number $2^{\text {ee }}$ then chair will move in forward direction then goes to room number 2 and likewise, when the user gives command like "Go to Back position" it moves towards back likewise "Left", "Right" and "Stop" for making the wheelchair to stop. This is designed for saving the cost, time and energy of the disabled persons. IR sensors are used and they help in detecting the obstacles in the passage.
\end{abstract}

Keywords: Arduino Uno, Wheelchair, Infrared sensor, Physically challenged, detect obstacles.

\section{INTRODUCTION}

An idea to make the people's life easier who are unable to walk by themselves due to accident, paralysis or age criteria. Most of the disabled persons depend on others for taking them wherever they want. The ones those who use wheel chair also have to depend on others for stirring it. They will always feel it difficult as they have to rely on others and cannot be independent. An Electrical wheelchair will give an independent feel for those who are with a physical disability or for those who use a mechanical wheelchair which requires help of others. But, still most of the people don't use smart wheel chair thinking that its costly.

The proposal in the paper describes simple, smart, affordable, key, motor controlled device which is friendly and customized commands are given and it will make the wheel chair move independently. Smart phone is used as the brain of the robot for giving the instructions. Bluetooth has made communication easier from wired to wireless. This is based on key and motor controlled device. IR sensors are also used for sensing and giving notification if it finds an obstacle in the passage.

\section{LITERATURE SURVEY}

Hymavath et al (2011) is the one who designed the program for the mobile robot that follows a dark line on the white background and detect turns or the deviations and to then to control the functions of the motors.

\footnotetext{
Revised Manuscript Received on August 14, 2019.

N.Monicka, Student, Department of ECE, Saveetha Institute of Medical and Technical Sciences, Chennai, Tamilnadu, India.(email: monickan156@gmail.com)

Bhuvaneswari Balachander, Assistant professor, Department of ECE, Saveetha Institute of Medical and Technical Sciences, Chennai, Tamilnadu,
} India.(email: bhuvaneswari.balachandar@gmail.com)
Lee et al. (2013) is the one who proposed that a robot has the capability to follow the line on the floor with two wheels using visual feedback.

Azlan et al (2007) is the one who described that an RCX LEGO robot incorporates an on-board Hitachi H8 microprocessor. The IR sensors were used under the robot to sense a line drawn whether it is white or black on a black surface. To make the robot to follow the line path fuzzy logic was used. Due to the scale of those robots, the process resources obtainable area unit quite restricted thus easier models and techniques, like visual servoing, area unit accustomed scale back the process.

Ibrahim et al (2009) developed a line follower from a tank bot used twin line sensors. They also designed a cost efficient microcontroller based robot which has a pair of IR reflectors attached at the bottom and at both the corners of the robot.

Su et al (2010) used the PID controller to make the robot follow the racing track and PD controller for reducing error between sensor 55 and track. Some systems area unit even capable of functioning, once given Associate in Nursing correct model of the atmosphere, mistreatment solely vary finding.

Budihatro et al. (2010) - For the humanoid robot he presented an Adaptive Neuro Fuzzy Inference System. On the track the robot follows a black line. Some problems have been rectified in this project. Electric wheelchair needs an IR guidance whereas line follower wheelchair is on. Manual shutdown and automatic shutdown depending on causion. Need to be focused while controlling its motion where as it is an free hand motion mechanism. Manual stop when obstacles appear where as it will automatically stop on obstacle detection.

\section{ARCHITECTURE}

The design consists of an Arduino, a Bluetooth, Motor Driver and a smart phone. The Bluetooth transmits the commands given. User gives the command to Arduino using Bluetooth. The Bluetooth is connected to arduino by installing an application in a smart phone. The instruction will be given to arduino. The microcontroller receives instruction and will make motors function. Wheelchair moves according to commands given. In mean time when circuit is ON the IR sensors senses and will make sure that there is no obstacle. If it detects then instruction will be sent and wheel chair will stop. 


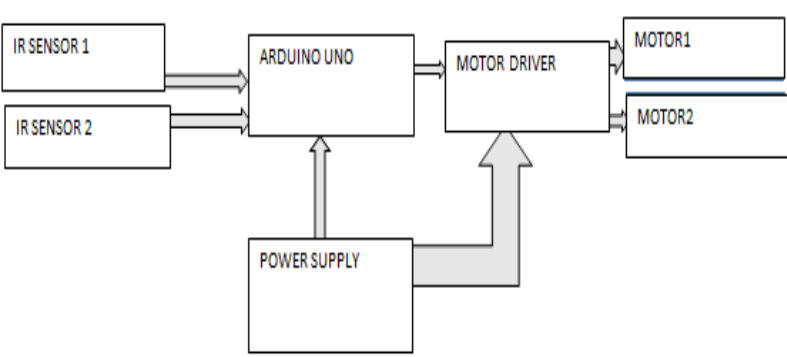

Figure 1: Block diagram for wheel chair

The IR sensors shown in Fig.1 senses the path and makes sure that there is no obstacle. The external power supply is given to the arduino where the programs are coded and it is also connected to the motor driver. The motors get external power supply from the motor driver.

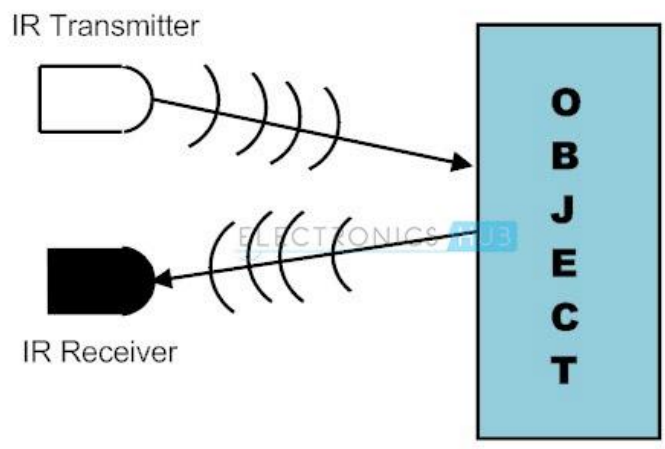

Figure 2: IR Sensor

IR sensors shown in Fig.2 transmit infrared light. It is based on a principle that when IR senses a white line it reflects back and when it senses a black line it doesnot reflect. The idea of an Infrared Device is to sense the obstacle and to transmit a signal that gets received.

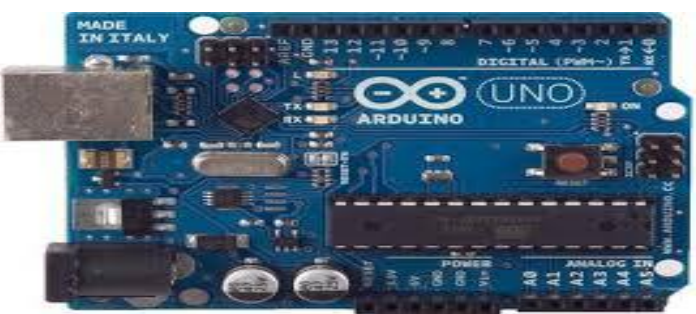

Figure 3: Arduino

The Arduino Uno as shown in Fig. 3 is a microcontroller board that has all instructions coded.

\section{Physical Characteristics of Arduino:}

The has power jack and USB. The panel is attached to the case by four screw holes. The other pins have 100 mil space but the pins 7 and 8 have 160 mil.

\section{Motor Driver:}

Motor drives are attached to motors and gives them external power supply.

\section{Power Supply Unit:}

Power supply unit gives the external power supply to the motors. Conversion of an electrical phenomenon from a supply to the proper voltage, proper current, and proper frequency to power the load is the main purpose.

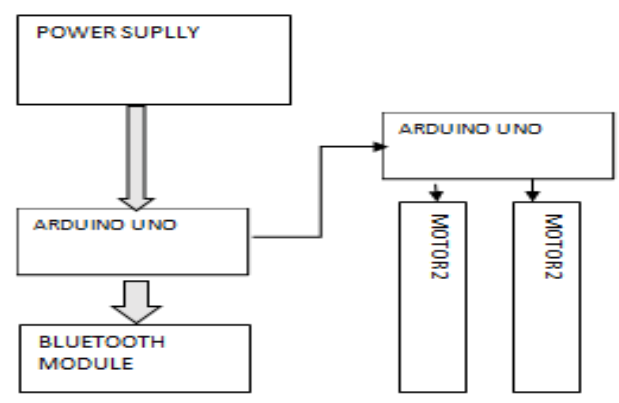

Figure 4: Block Diagram for wheel chair's path

Block diagram for smart wheel chair's path is represented in fig.5. Then the arduino is connected to the which is linked to a smart phone. Then the arduino is again connected to the motors. Through the software application which is installed in the smart phone the instructions will be given and the Bluetooth receives the instructions and function accordingly.

\section{Bluetooth Module:}

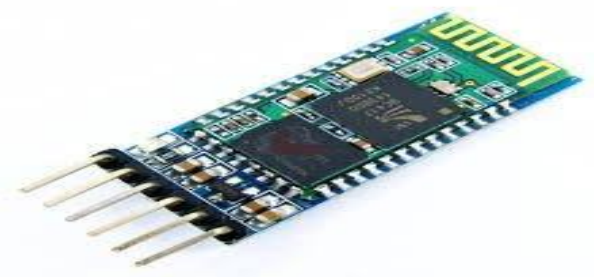

Figure 5: Bluetooth Module

Bluetooth Module shown in Fig 6 gets the instruction from the software application installed and it makes the device to function according to the commands programmed in the arduino.

\section{APPLICATION INSTRUCTIONS}

Different types of motions like front, back, right, left and stop are possible.

1. Firstly the Bluetooth must be paired with the android phone that has the software application installed.

2. When the user gives instruction like"go to room number 3", Bluetooth Electronics application sends the command. Command is received and the motors make the wheelchair move forward.

3. When the user gives instruction like" back to room number 1" Bluetooth Electronics application sends the command. Command is received and the motors make the wheel chair move backward.

4. When the user gives left key command then the Bluetooth Electronics application sends the command. Command is received and the motors make the wheel chair move left.

5. When the user gives right key command then the Bluetooth Electronics application sends the command. Command is received and the motors make the wheel chair move right.

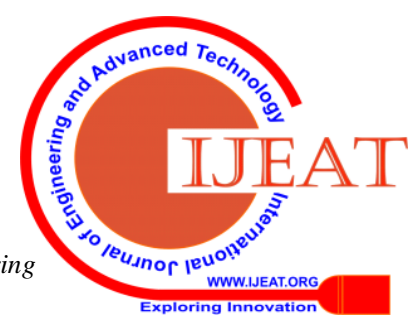


6. When the user gives stop key command then the Bluetooth Electronics application sends command to the module connected to the circuit. The instruction will be received and the motors will stop the motion of the wheel chair.

When you click on the key for disconnecting then it gets unpaired.

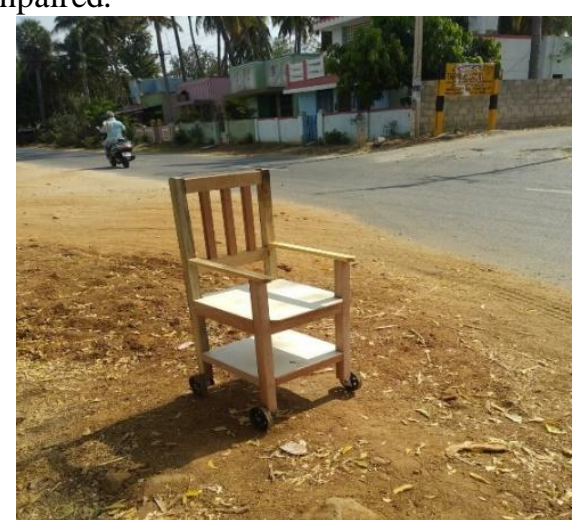

Figure 6: Wheel chair Design
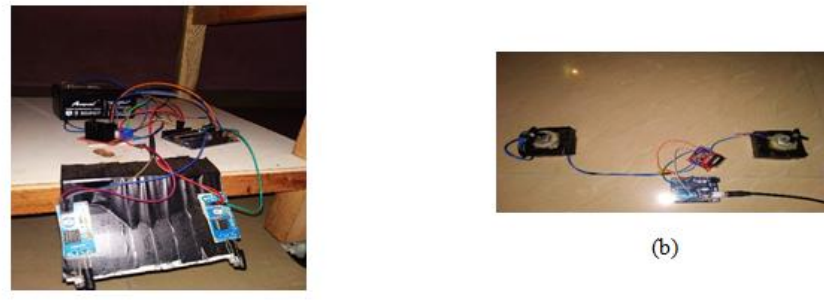

(b)

(a)

Figure 7 (a) (b) : Assembly of the electronic components

The hardware design consists of the assembly of electronic components as shown in fig.7(a)(b). The commands of the user are given to the system by the help of the Bluetooth it is sent to the device. According to commands given the motors make wheel chair move.

\section{EXPERIMENTAL RESULTS}

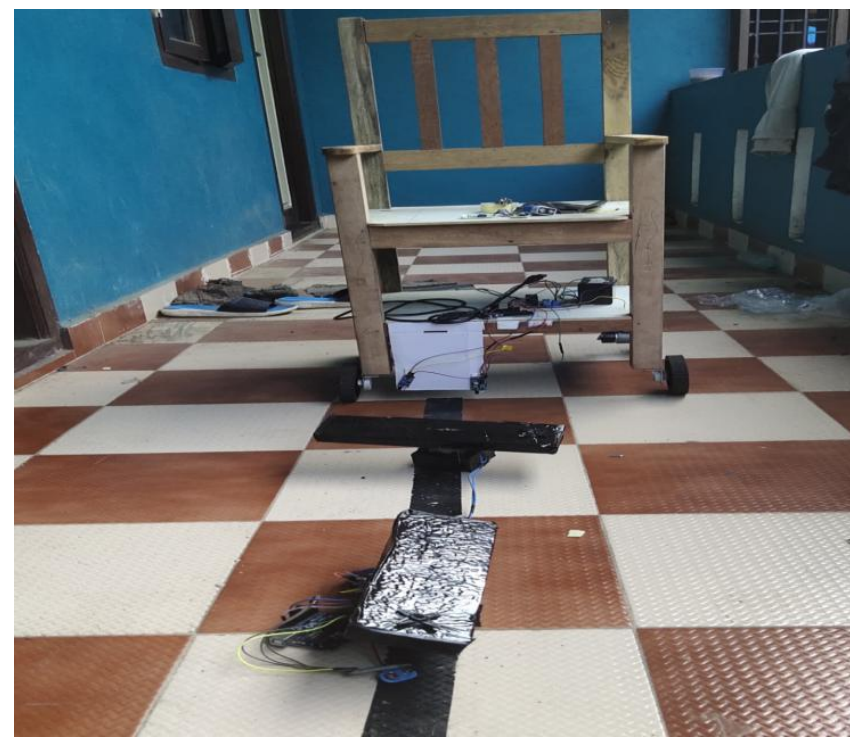

Figure 8: Output of the wheel chair with its path

The final output of the wheel chair with its path is shown in fig.8. For example, when the user gives command like "Go to room number $2^{\text {ee }}$ then chair will move in forward direction then goes to room number 2 and likewise, "Go to Back position" the chair moves towards back ,likewise "Leftec, "Right" and "Stop" for making the wheelchair to stop.

\section{VI .CONCLUSION}

Paper explains design and execution of an wheel chair using Bluetooth. The wheel chair follows the commands given. After design process of the circuit it has been tested to check whether it follows the command of the user and moves correctly. It also senses the obstacle which is lying in its path. The obstacle is sensed by the IR sensors. This project will help the differently abled persons and the aged people to live independently.

\section{REFERENCES}

1. Azam, G., and M. T. Islam. "Design and Fabrication of a Voice Controlled Wheelchair for Physically Disabled People"

2. Anusha, S., M. Madhavi, and R. Hemalatha. "HOME AUTOMATION USING ATmega328 MICROCONTROLLER AND ANDROID APPLICATION." (2015).

3. Rajini, Gangadhari and Lr Siva. "Android Mobile Phone Controlled Bluetooth Robot Using Arm7 Microcontroller." (2015).

4. Megalingam, Rajesh Kannan et al. "'Gest-BOT'-A Highly Convenient Locomotive Solution for the Elderly and Physically Challenged." Global Humanitarian Technology Conference (GHTC), 2012 IEEE. IEEE, 2012.

5. Skraba, Andrej, et al. "Prototype of speech controlled cloud based wheelchair platform for disabled persons." Embedded Computing (MECO), 2014 3rd Mediterranean Conference

on. IEEE, 2014

6. M. Carmel, V. Brindha, and A. Abudhahir. "Facial expression recognition using PCA based interface for wheelchair." Electronics and Communication Systems (ICECS), 2014 International Conference on. IEEE, 2014.

7. Klabi I., Masmoudi M.S., Masmoudi M., "Advanced user interfaces for intelligent wheelchair system", 1st IEEE Conference on Advanced Technologies for Signal and Image Processing, 2014, pp.130-136, Tunisia.

8. L. Javier and J. Y. Zhou, Wireless Sensor Network Security, vol. 1, IOS Press, 2008.

9. Moghavvemi, M., K., C. Y. Soo, and S. Y. Tan.2005."A reliable and feasible remote sensing system for temperature and humidity measurement. "Sensors and Actuators A 117:181-185.

10. Kuruvadi Praveen, Ankhit BalaVenkata "Modular Weather and Environment Monitoring Systems using Raspberry $\mathrm{Pi}^{\mathrm{ee}}$,International Journal of Engineering Research \& Technology, (IJERT), Vol. 3 Issue 9, September- 2014. 